\title{
HARMONISING BUSINESS PROCESSES OF COLLABORATIVE NETWORKED ORGANISATIONS USING PROCESS MODELLING
}

\author{
John Krogstie \\ SINTEF and IDI, NTNU, NORWAY. John.Krogstie@sintef.no \\ Vibeke Dalberg, Siri Moe Jensen \\ Det Norske Veritas, NORWAY. \{Vibeke.Dalberg,Siri.Jensen\}@dnv.com
}

\begin{abstract}
Process modelling is often used in connection to the introduction of standardised information systems in traditional organisational forms. Existing work on success measures of process modelling has summarised important aspects In this setting.

In this paper we present an investigation into process modelling success in connection to harmonising the work across units that has traditionally been to a large extent autonomous. We present a pilot case from a global serviceproviding enterprise, from which we have tried to extract the value of process modelling in a networked organisation, comparing to existing frameworks of process modelling success. Although many of ourfindings are supported by existing frameworks, we have found a need to combine and extend these to better grasp the value of process modelling in connection to harmonising more loosely integrated organizational forms.
\end{abstract}

\section{INTRODUCTION}

The modelling of work processes, organisational structures and infrastructure as an approach to organisational and software development and documentation is becoming an established practice in many companies. This development is also noticeable in our case organisation, a global service-providing company delivering a broad range of classification, certification, and consulting services worldwide.

According to Sedera, Rosemann, and Doebeli (2003), who address best practices for a post-hoc evaluation of process modelling activities, a modelling project can be regarded as successful if it is efficient and effective. Model quality in various domains is being addressed by Krogstie (2003), Moody (1998), and Waal (2002). Kawalek (1997) links problem and success areas in connection to process modelling to a traditional project lifecycle.

The above-mentioned discussions and evaluations of value is limited to single projects in a stable organisational situation without directly taking into account 
aspects specific of more complex organisational forms such as networked organisations. In previous work (Krogstie, 2002) we have identified main challenges for networked organisations, all that can be supported or influenced with process technologies:

In this paper we will use and extend existing frameworks to assess success of a modelling initiative in this setting. To this end we use a pilot case reported on the following pages. After presenting the case, section 3 describes our research method. Main results are described in section 4, before we sum up in section 5 .

\section{BACKGROUND ON CASE STUDY}

The case organisation was established 150 years ago as a maritime classification organisation. New offices were over the years established around the world to meet customers need. The business rules and work procedures were established centrally, and applied locally in the new offices around the world. Around 20 years ago, small independent initiatives on providing certification services started up in some of these offices. The certification activity within the different units soon increased, and the business area Certification was established. In contrast to the maritime industries within the organisation, Certification was not initiated from the main office, but each certification unit was developed based on local needs and procedures. Each certification unit developed their own systems and work procedures for back-office and support activities, such as marketing, sales, planning, issuing certificates etc. Some units also developed their own software applications. From the mid 1990's a common software application was locally implemented in some units. Over the years each unit personalised this tool to their local needs. In 2000 Certification centrally decided to focus on improved efficiency, and chose to start the work on harmonising the work processes. The harmonised work processes should be standardised processes, with the possibilities of local adaptation. This way, Certification is now implementing centrally based processes, in a business that is used to work according to local procedures. A new software application to support the harmonised work processes is currently being implemented throughout the organisation.

The project has actively used modelling, both in developing the harmonised processes, and in communicating them to different types of audience. The project has reused knowledge as well as models from earlier process model activities within the business area, and the harmonised work process models are also being further used in other, later initiated projects within the organisation.

The work processes within the business area were first modelled at a high level in the mid 1990's as a part of an official document describing the business. These models were created in a spreadsheet (Excel), but have later been implemented in the modelling tool Visio using IDEF0 process modelling.

In 2000 it was decided to look into possible changes in the work processes within the business area, how these were developed and adapted locally, and what could be improved. A pre-project went through several workshops involving partners from different offices within the network, where domain experts from the business area worldwide defined a list of areas to improve in the current work processes. The pre-project concluded on starting an efficiency and harmonising 
project, in order to first develop an ideal process, and then a harmonised process, and also looked into the possibility of information system support.

The 'ideal' work processes was described. When defining the harmonised process, technical and economical constraints have influenced the models. This modelling was performed by the process developer, and the target audience of the models were domain experts. The results of this process were textual descriptions and high-level models of the harmonised processes.

An IS-development project was established in 2001. The process models were initially used by the process developer to communicate with the software engineers. The process developer later modelled the work processes on a detailed level using swimlanes (a variant of Role-Activity Diagrams (Ould, 1995)).

In order for the harmonised processes to be implemented worldwide, key personnel from the business area management went to the different local units analysing their local work processes in relation to the harmonised processes (gap analysis). The requirements to the units were that the new information system would not be implemented in the unit before the gaps were closed. The IDEF0 models of the harmonised processes were actively used during the analysis process.

\section{RESEARCH METHOD}

The research presented in this paper is based on qualitative analysis of a case study. According to Benbasat, Goldstein, and Mead (1987), a case study is well suited when the context of investigation takes place over time, is a complex process involving multiple actors, and is influenced by events that happen unexpectedly. Our case satisfies these criteria. Yin (1994) states that the relevance of a single case study is high when the goal is to identify new and previously not researched issues. We have wanted to identify the value of modelling in world-wide organisation of partly autonomous units. There has not been reported much research within this area earlier, thus we have chosen a single case approach.

Based on input from a preliminary series of interviews and literature studies, an interview guide was established. These interviews were focused on expected and experienced use and value from the modelling efforts in the case study, aiming at identifying as many expectations as possible, including any that may not have been documented in project documentation because they were not considered directly relevant for the project goal. After initial open questions, the interviews were structured around keywords from the work of Sedera et al (2003) on process modelling success.

Documentation of the study is based on these interviews, studies of project documentation and models. The interview guides where used as basis for structuring contact summary sheets with the main concepts, themes, issues and questions relating to the contact (Miles and Huberman, 1994). We used conceptual modelling techniques (Krogstie, 2003) to structure this information, with a focus on the expectations, modelling tasks, modelling projects, roles, modelling concepts, tools involved, and experiences. These concepts were used as a basis for coding the data. Relationships between the identified concepts gave a basis for pattern coding. Case analysis meetings where held regularly between the researchers. The final article 
was reviewed by the involved parties, and further input was added by the project manager of the efficiency and harmonising project.

\section{RESULTS AND DISCUSSION}

Sedera et al (2003) have presented a process modelling success model where the identified success measures in their model are:

1. Model-use: How extensively the models are applied and utilised.

2. User satisfaction: The extent to which users believe process modelling meets the fulfilment of the objectives that underlay the modelling project.

3. Process impact: Measures the effects of modelling on the process' performance.

4. Process model quality: The extent to which all desirable properties of a model are fulfilled to satisfy the needs of the model users.

Trying to use this framework, we quickly discovered that their discussion is to a large extent based on modelling in connection to the development of ERP-systems in a traditional organisation, and that in our case, a more detailed model was needed to explain all the relevant aspects for a networked organisation. We will below discuss and extend each of the areas of their model, relating it to the case.

\subsection{Model use}

Models can be used for a number of different purposes. According to Curtis, Kellner, and Over (1992), Totland (1997), and Vernadat (1996), enterprise process models may be usefully utilised in the following areas:

1. Human-sense making and communication to make sense of aspects of an enterprise and to communicate with other people.

2. Computer-assisted analysis to gain knowledge about the enterprise through simulation or deduction.

3. Model deployment and activation to integrate the model in an information system and thereby actively take part in the work performed by the organisation.

4. The model is a basis and gives the context for a system development project, without being directly implemented (as is done in 3).

An orthogonal dimension to these four is the temporal dimension, i.e. if one are to model the past, the present (as-is) or the future (to-be). Another key differentiator is to what extent the focus is on internal processes to a company, or to support interorganisational co-operation. Finally one can differentiate between process models on a type level and on an instance layers.

The IDEF0 models in our case study have been used for communication for different purposes within the business area (usage area 1). Examples are the use of the models as a communication artefact between domain experts in order to improve the work processes, or the use as an input to the requirement specification of the information systems development project.

The swimlane models were used for the software developers to understand the domain, and during IS development (vs. usage area 4), but needed to be adapted quite a bit to be useful at this level. The IDEF0 models were used for developing the 
user interfaces of the information system. The work process models were reflected through the menu-structure in the information system, and in this way there is a direct link between the models and the information system.

The IDEF0 models were also used as a tool to perform the initial gap analysis in the units. For rollout purposes, the modified swimlanes were used in order to both reveal the gaps, and in training the users. The process models were also used when discussing with units that hesitated to change their current processes.

During the project, the IDEF0 models, which gave an overview of the new work processes, were used satisfactory for high level discussions, while they were not sufficient when details and specific information was needed. This was specifically evident in the initial gap analysis in the different units.

\subsection{User satisfaction}

There are several different user categories of the models, not only one. There can possibly also be unidentified users. Examples of users are domain experts, workers, end users, super-users, management including the project sponsor, process modeller (external e.g. a consultant, and internal), process owner, and IS developer.

This is even further complicated by the aspects related to that the users was spread across a number of different partly autonomous units, which was organising their work quite differently based on cultural and other issues, such as unit size.

The users of the IDEF0 models were divided in their level of satisfaction of the models, depending on their needs. The domain experts using them in discussions on the harmonised process seemed satisfied with the models and what they could offer in their setting. The IS developers did not find these models satisfactory, because they did not teach them about the domain, and they were not detailed enough to tell them about the requirements of the information system to be developed. The swimlanes were to much more help during the development, but were not totally satisfactory due to problems with keeping models from several modelling tools and with varying levels of detail consistently updated.

The overall impression of the process developer was that end users have reacted positively to having their new work processes presented visually in the form of models. Project management also seems satisfied with the model on an overall level. More work could have been done to involve the different users more actively in the modelling up-front, using a more user-centred design approach (ISO, 1999).

\subsection{Process impact}

There are several different processes that can be impacted e.g.

- The process itself of developing the harmonised process.

- The model's effect on creating the harmonised process.

- The previous work processes in each local unit.

- The harmonised work process.

- The new work processes in each local unit.

- The process of creating the application.

- The process of implementing the application in the organisation.

The consequences of implementing the harmonised processes are that all units have to change their work processes somewhat, supported by a new information 
system. One informant claimed that it was not the models that changed the work processes in the unit, but the process of performing the modelling. The models acted as a very important artefact to focus the discussions around.

The representative of the IT department claimed that the process models had created "a fantastic foundation for the participants in the project", and that they were important to be able to change the work processes in the business area by establishing a common understanding across the different units.

Issues that do not fit into the model used as a communication artefact are left out of the conversation, the process modeller claimed. To not influence the explanation of the work processes by the units, the models were only used as a checklist by the rollout responsible. Then, in the next meeting he used the swimlanes in the training of and discussion with the units, focusing on the areas that did not fit with the harmonised process. In this way, the models have changed the rollout process within the project because they were found to have a too strong effect on the participants, hindering the identification of gaps. In future work we will investigate and evaluate in more detail hypothesis about cost-benefit and reduction of risks in the process.

\subsection{Process model quality}

The focus on quality of the process models differ from user group to user group. The Visio harmonised process models were initially created to act as a communication artefact among domain experts, and their needs were fulfilled by representing the models on a high level, but detailed enough to discuss and agree on the new processes. The Visio harmonised process models did not satisfy the needs of the software developers or, identified at a later point, the needs of a gap analysis performed at the individual units. The models were for some time not updated, and this lowers the quality of them. Model quality can be looked upon across a number of levels (Krogstie and Sølvberg, 2003). Some aspects already mentioned above will be stated also here if they fit this framework.

Physical quality: The basic quality goals on the physical level are externalisation, meaning that the knowledge of the domain of some social actor has been externalised by the use of a modelling language, and internalisability, that the externalised model is persistent and available, enabling the audience to make sense of it. It is obviously important that the models were externalised, but the amount of detail necessary differed between different usage areas as discussed above. As for software development, the amount of detail was too small, and not possible to easily add to the existing models, due to both the availability of resources and the limitation of the chosen modelling tool.

Empirical quality deals with predicable error frequencies when a model is read or written by different users, and coding (e.g. shapes of boxes). One example here is to limit the number of crossing lines in a model. This aspect was important in connection to the task of creating a common overall picture which could be adapted across all different units. The high-level model view was successful for this. Also the swimlane view of the detailed processes was good in connection to the matching of the harmonised model with local processes when used in the right way.

Syntactic quality is the correspondence between the model and the language in which the model is written. This is typically evaluated through syntax checking. 
Several syntactic errors are found in all the models. This has not been regarded as important for the main uses of the models so far.

Semantic quality is the correspondence between the model and the domain. The quality framework contains two semantic goals; validity (all statements in the model are correct relative to the domain), and completeness (the model contains all the relevant statements which is found in the domain). It has been difficult to keep the models up to date and internally consistent, due to resource and tool limitations. This has spawned the need for improved tool-support. Many areas within the overall process model has not been modelled in detail. One specific aspect in a case like this is the need to develop a harmonised model, i.e. that the model can be locally adapted due to heterogeneous IS infrastructure, different quality control procedures and local cultures. Thus, the model is really not right or wrong, but rather more or less appropriate for the different units. To act as an integrating artefact though, e.g. to support communication and learning, focus on a common model is important.

Perceived semantic quality is the similar correspondence between the audience interpretation of a model and his or hers current knowledge of the domain, and is what can actually be evaluated at quality control and validation. For certain tasks, the model has been perceived to be better than it actually is. One example is the gap analysis where the gaps were not discovered early enough.

Pragmatic quality is the correspondence between the model and the audience's interpretation of it. It is differentiated between social pragmatic quality (to what extent people understand the models) and technical pragmatic quality (to what extent tools can be made to exploit the model). Comprehension of the high-level model appeared to be good, partly because people were able to put their own meaning into them. The more detailed the models were, the more difficult it became to understand for others than those working with them on a daily basis. As for technical pragmatic quality, this has so far been missing.

Social quality is focussed on agreement among audience members' interpretations. This has been very good for the high-level model, which is also the area were high social quality is most important. The process and model developer explained that due to the fact that the detail level of the models during the gap analysis, everyone could agree on the new processes, which again created a loyalty to the processes and solidarity within the overall certification business area. One informant claimed that the project was truly dependent upon this, and that it has helped the process of implementing the new harmonised processes to a great extent.

It is interesting to note how the use of the models for different goals and modelling tasks shifts the emphasis on model quality. To create a common vision across the partly autonomous units (the high-level Visio harmonised process models), it is very important that the model is empirically good ("looks nice"), and is socially agreed, whereas syntactic correctness and semantic completeness is usually only of minor importance. For the use towards IS-developers on the other hand, semantic completeness was an important issue. In connection to gap analysis it was in fact important that the models were not shown at first, since they had very high perceived semantic quality in the sense that they were apparently accepted at face value, without being questioned. This is an interesting example of the model monopoly problem (Bråten, 1984). This also highlights a major weakness of the original framework of Sedera et al. Their dimensions are not orthogonal. Model use 
and types of users influence how to look upon process model quality, and which quality aspects to emphasize.

\section{CONCLUSION}

We have in this paper applied and extended a framework for process modelling success related to a project where the goal has been to harmonise a networked organisation of partly autonomous organisational units.

Whereas most aspects found can be discussed within the broad areas defined by Sedera et al., we also recognise the need to deepen their categories for the use of the framework to be meaningful, and that their dimensions are not orthogonal.

Whereas many of the extensions could also be useful in a more traditional modelling project, several dimensions are related to that the model is used to harmonise different units within a virtual organisation, where the different background and context of the different units necessitate the support of a harmonised rather than a standardised model as highlighted under the discussion on semantic quality.

In future work we will investigate and evaluate in more detail hypothesis about cost-benefit, performance, and reduction of risks in the process and use this to device an improved methodology for this kind of work.

\section{REFERENCES}

1. Benbasat, I., Goldstein, D. K. and Mead, M. "The case research strategy in studies of informations systems" MIS Quarterly (11:3) 1987, p 369-386.

2. Bråten, S. Dialogens vilkår i datasamfunnet (in Norwegian). Oslo, Universitetsforlaget, 1983

3. Curtis, B., Kellner, M., Over, J. "Process Modelling," CACM, (35:9), September 1992, pp. 75-90.

4. EN ISO 13407 "User-centered design process for interactive systems", 1999.

5. Kawalek, P and Kueng, P. The usefulness of Process Models: A Lifecycle Description of how Process Models are used in Modern Organization. EMMSAD'97 Pisa Italy

6. Krogstie, J., Jørgensen, H. D., and Lillehagen, F. Active models for digitally enabled creative business networks, IFIP World Computing Conference. Montreal, Canada, August 2002.

7. Krogstie, J. and Sølvberg, A. Information Systems Engineering: Conceptual Modeling in a Quality Perspective, Kompendiumforlaget, Trondheim, Norway, 2003.

8. Miles, M. B, and Huberman, A. M. Qualitative Data Analysis, SAGE Publications 1994.

9. Moody, D. L. Metrics for Evaluating the Quality of entity-relationship models. Proceeding of ER'98 Singapore, LNCS 1507, Springer-Verlag, 1998.

10. Ould, M.A. Business Processes - Modeling and Analysis for Re-engineering and Improvement. John Wiley \& Sons, 1995.

11. Sedera, W., Rosemann, M. and Doebeli, G. "A Process Modelling Success Model: Insights From A Case Study". 11th European Conference on Information Systems, Naples, Italy, 2003.

12. Totland, T. Enterprise Modelling as a means to support human sense-making and communication in organizations. IDI. Trondheim, NTNU. 1997.

13. Yin. R. Case study Research. SAGE Publications. 1994

14. Vernadat, F. Enterprise Modelling and Integration. Chapman and Hall. 1996.

15. Waal, A. L. Quality of Enterprise Models and Enterprise Modelling. Project report, IDI, NTNU, Trondheim, Norway. 2002. 